Reiner Dumke

Modernes

Software Engineering 
vieweg

Lehrbuch Informatik

Aufbau und Arbeitsweise von Rechenanlagen von Wolfgang Coy

Mehr als nur Programmieren... Eine Einführung in die Informatik von Rainer Gmehlich und Heinrich Rust

Computergrafik in der Differentialgeometrie von Eberhard Malkowsky und Wolfgang Nickel herausgegeben von Kurt Endl

\section{Modernes Software Engineering}

Eine Einführung

von Reiner Dumke

Modellbildung und Simulation

von Hartmut Bossel

Softwareentwicklung nach Maß

von Reiner Dumke

Parallele Programmierung

von Thomas Bräunl

„Elemente“ der Informatik

Ausgewählte mathematische Grundlagen

für Informatiker und Wirtschaftsinformatiker

von Rainer Beedgen

Computersicherheit

von Rolf Oppliger

Formale Methoden und kleine Systeme von Dirk Siefkes

Numerik sehen und verstehen

von Manfred Bretz 
Reiner Dumke

\section{Modernes \\ Software Engineering}

Eine Einführung 
Das in diesem Buch enthaltene Programm-Material ist mit keiner Verpflichtung oder Garantie irgendeiner Art verbunden. Der Autor und der Verlag übernehmen infolgedessen keine Verantwortung und werden keine daraus folgende oder sonstige Haftung übernehmen, die auf irgendeine Art aus der Benutzung dieses Programm-Materials oder Teilen davon entsteht.

Alle Rechte vorbehalten

(C) Friedr. Vieweg \& Sohn Verlagsgesellschaft mbH, Braunschweig/Wiesbaden, 1993

Der Verlag Vieweg ist ein Unternehmen der Verlagsgruppe Bertelsmann International.

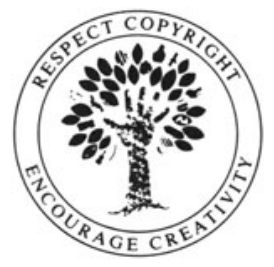

Das Werk einschließlich aller seiner Teile ist urheberrechtlich geschützt. Jede Verwertung außerhalb der engen Grenzen des Urheberrechtsgesetzes ist ohne Zustimmung des Verlags unzulässig und strafbar. Das gilt insbesondere für Vervielfältigungen, Übersetzungen, Mikroverfilmungen und die Einspeicherung und Verarbeitung in elektronischen Systemen.

Gedruckt auf säurefreiem Papier 


\section{Vorwort}

Das Software Engineering (Software-Technik bzw. Software-Technologie) hat sich inzwischen zu einem sehr umfangreichen Fachgebiet der Informatik entwickelt. Es versucht, für die verschiedendsten Software-Entwicklungsprozesse geeignete Mittel und Methoden zur Verfügung zu stellen. Die heutige Software-Entwicklung ist vor allem gekennzeichnet durch

- eine zunehmende Komplexität der zu realisierenden Systeme,

- eine zunehmende Parallelisierung der implementierten Problemstellungen,

- eine wachsende Qualitätsanforderung an die laufenden SoftwareProdukte und durch

- erhöhte Sicherheitsanforderungen sowohl hinsichtlich Zuverlässigkeit als auch Berechtigungsabsicherung.

Die auf dem Gebiet des Software Engineering vorhandene Literatur ist ebenso vielfältig aber größtenteils speziellen Aspekten gewidmet. Das vorliegende Lehrbuch zum Software Engineering entstand auf der Grundlage langjähriger Erfahrung bei der Planung, Leitung und der Realisierung umfangreicher Software-Projekte sowie der langjährigen Ausbildung von Informatikstudenten im Fach Software-Technik. Es dient vor allem

- den Studenten der Universitäten und Fachhochschulen als einführendes Lehrbuch in die Probleme des Software Engineering,

- der Vermittlung von Grundkenntnissen zum Software Engineering, die den Leser in die Lage versetzen sollen, auf der Grundlage der zu jedem Abschnitt angegebenen Literatur, sich tiefer in ein Spezialgebiet einarbeiten zu können,

- als Nachschlagewerk für bereits in der praktischen SoftwareEntwicklung tätige Programmierer und Software-Entwickler überhaupt.

Es setzt dabei Grundkenntnisse in der Programmierung und zum geringen Teil auch aus speziellen Gebieten der Mathematik, wie der Mengenlehre, der Graphentheorie und der Statistik, voraus. Der Titel ,Modernes Software Engineering" wurde aus folgenden Gründen gewählt: 
1. Die modernen Software-Entwicklungsmethoden sind auf neue Anwendungsgebiete gerichtet und versuchen dabei neue, vor allem effektivere Techniken zu verwenden. Dazu zählen beispielsweise die durchgängige objektorientierte Software-Entwicklung, die formale Spezifikation und die damit einhergehende Qualitätsverbesserung der SoftwareProdukte.

2. Bei der Entwicklung moderner Software-Produktionsverfahren werden häufig die Erekenntnisse aus, ,klassischen" Paradigmen nicht beachtet. Deshalb sind für das Verständnis und der höheren Effektivität künftiger Software-Entwicklung auch Kenntnisse aus diesem Bereich erforderlich.

3. Die Entscheidung über die Qualität und die Effektivität von SoftwareEntwicklungsmethoden setzt genaue quantifizierte Kenntnisse voraus. Daher ist die Software-Messung von Anfang an in die SoftwareEntwicklung mit einzubeziehen und auch Gegenstand dieses Buches.

Nach einem einleitenden Kapitel beschäftigt sich das zweite Kapitel mit dem Programmieren im Kleinen und behandelt ausgewählte Programmierungsund Software-Entwicklungsgrundlagen aus diesem Bereich. Der Programmierung im Großen widmet sich das dritte Kapitel. Neben den vielfältigen Problemen einer Software-Produktion und einigen ausgewählten klassischen Entwicklungsmethoden geht es hierbei um moderne Verfahren, wie die formale Spezifikation und die CASE-Tool-gestützte objektorientierte Software-Entwicklung. Übungsaufgaben zu den einzelnen Abschnitten und einige Lösungshinweise am Ende dieses Buches ermöglichen eine selbständige Einarbeitung in die verschiedenen Bereiche des Software Engineering.

Meinen Dank für zahlreiche Hinweise und Anregungen zu den einzelnen Themenbereichen gilt Frau Prof. Floyd, den Herren Prof. Stuchlik, Dr. Zuse, Dr. Winkler und Herrn Ulrich, sowie meinen Kollegen für die Unterstützung bei der redaktionellen Bearbeitung. Mein besonderer Dank gilt meiner Frau für ihr Verständnis und die Hilfe bei der Korrektur des Manuskriptes sowie dem Vieweg-Verlag für die entgegenkommende Zusammenarbeit. 


\section{Inhaltsverzeichnis}

1 Einführung 1

1.1 Der Begriff des Software Engineering . . . . . . . . . . . . . . 1

1.2 Gegenstand des Software Engineering . . . . . . . . . . . 3

1.3 Quellen- und Literaturhinweise . . . . . . . . . . . . . . . . 5

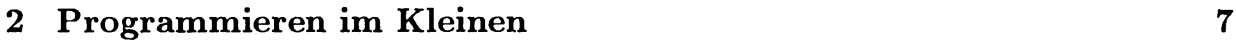

2.1 Einleitende Bemerkungen . . . . . . . . . . . . . . . 7

2.2 Strukturierte Programmierung $\ldots \ldots \ldots \ldots$

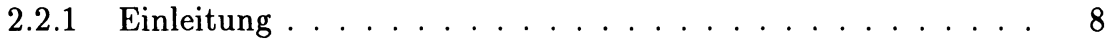

2.2 .2 Grundstrukturen der SP . . . . . . . . . . . . . . . . 9

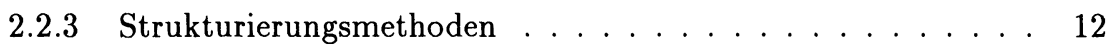

2.2.4 Der Pseudocode . . . . . . . . . . . . . . . . . 15

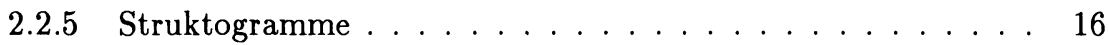

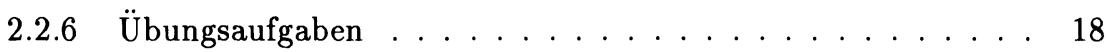

2.2.7 Quellen- und Literaturhinweise . . . . . . . . . . . . 18

2.3 Modularisierung . . . . . . . . . . . . . . . . . . . 20

2.3.1 Der Begriff des Moduls . . . . . . . . . . . . . . 20

2.3.2 Prinzipien der Modularisierung . . . . . . . . . . . . 20

2.3.3 Entwurfsarten . . . . . . . . . . . . . . . . . . . . . . . . 23

2.3.4 Ein Modularisierungsbeispiel . . . . . . . . . . . . . 24

2.3 .5 Übungsaufgaben . . . . . . . . . . . . . . . . . . 25

2.3.6 Quellen- und Literaturhinweise . . . . . . . . . . . 26 
2.4 Allgemeine Makroprogrammierung . . . . . . . . . . . . . . . 28

2.4.1 Grundbegriffe der Makrotechnik . . . . . . . . . . . 28

2.4.1.1 Der Makrobegriff . . . . . . . . . . . . . . 28

2.4.1.2 Makrosprachen ... . . . . . . . . . . 29

2.4.1.3 Makroprozessoren ... . . . . . . . . 30

2.4.1.4 Anwendungsgebiete ... . . . . . . . . 31

2.4.2 Spezielle Makrosprachen . . . . . . . . . . . . . . 31

2.4.2.1 Der C-Preprozessor ............ . 31

2.4.2.2 Das SUPERMAC-Prinzip . . . . . . . . . . . . 34

2.4 .3 Übungsaufgaben . . . . . . . . . . . . . . . . . . 37

2.4.4 Quellen- und Literaturhinweise . . . . . . . . . . . . . 37

2.5 Programmtest . . . . . . . . . . . . . . . . . . . . 39

2.5.1 Einleitung . . . . . . . . . . . . . . . 39

2.5.2 Statischer Programmtest . . . . . . . . . . . . . 40

2.5.2.1 Checklistenverfahren . . . . . . . . . . . . 40

2.5.2.2 Code-Reviews .............. . 41

2.5.2.3 Programmverifikation ... . . . . . . . 41

2.5.2.4 Symbolische Programmtestung ... . . . . . . 45

2.5.3 Dynamisches Testen ... . . . . . . . . . . . 45

2.5.3.1 Auswahl der Testdaten . . . . . . . . . . . 46

2.5.3.2 Allgemeine Testmethoden . . . . . . . . . . . 47

2.5.3.3 Der Black-Box-Test . . . . . . . . . . . . . 48

2.5.3.4 Der White-Box-Test ............. 50

2.5.3.5 Testhilfen ................ 54

2.5 .4 Übungsaufgaben ... . . . . . . . . . . . . . 55

2.5.5 Quellen- und Literaturhinweise . . . . . . . . . . . . 56

2.6 Programmodifikation . . . . . . . . . . . . . 58

2.6.1 Einleitung . . . . . . . . . . . . . 58

2.6.2 Programmverständnis . . . . . . . . . . . 58

2.6.2.1 Lesbarkeitsverbesserungen . . . . . . . . . . . 59

2.6.2.2 Programmierkonventionen ... . . . . . . 62 
2.6.2.3 Programmskelette . . . . . . . . . . . . 63

2.6.3 Programmodifikationsformen . . . . . . . . . . 65

2.6.3.1 Wiederverwendung . . . . . . . . . . . . 65

2.6.3.2 Transformationen . . . . . . . . . . . 67

2.6.4 Spezielle Programmcharakteristika . . . . . . . . . . . 72

2.6.4.1 Programmqualität . . . . . . . . . . 73

2.6.4.2 Programmversionen . . . . . . . . . 75

2.6.5 Übungsaufgaben $\ldots \ldots \ldots \ldots \ldots \ldots \ldots$

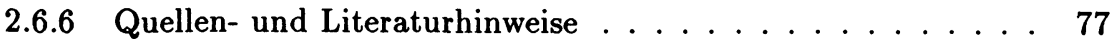

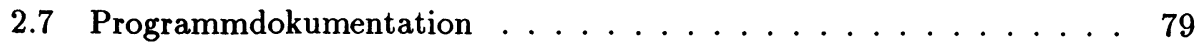

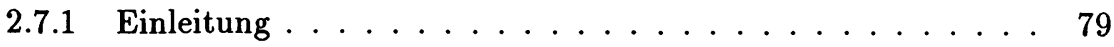

2.7.2 Literate Programming . . . . . . . . . . . . . . . 81

2.7.2.1 Das WEB-System . . . . . . . . . . . . . 81

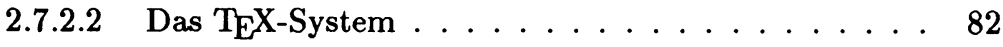

2.7.2.3 Das Makropaket $\mathrm{IAT}_{\mathrm{E}} \mathrm{X} \ldots \ldots \ldots \ldots . \ldots . \ldots 85$

2.7.2.4 Das Pascal-WEB-System . . . . . . . . . . . 89

2.7.2.5 Das Cweb-System . . . . . . . . . . . . . 93

2.7.3 Übungsaufgaben . . . . . . . . . . . . . . . . . 94

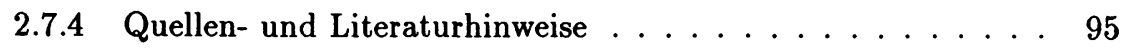

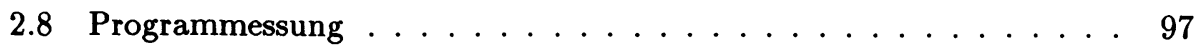

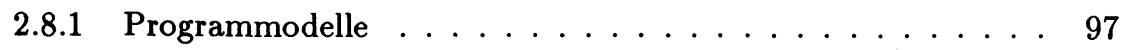

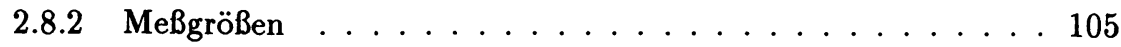

2.8.2.1 Das Leistungsverhalten . . . . . . . . . . 105

2.8.2.2 Die Programmkomplexität . . . . . . . . . . 105

2.8.3 Maßbeispiele . . . . . . . . . . . . . . . 108

2.8.3.1 Lines Of Code . . . . . . . . . . . . . . . . . . 109

2.8.3.2 Halstead's Software Science . . . . . . . . . . . . 111

2.8.3.3 Das McCabe-Maß . . . . . . . . . . . . . . . 115

2.8.3.4 Das Henry/Kafura-Maß . . . . . . . . . . . . . 116

2.8.3.5 Das Blaschek-MaB . . . . . . . . . . . . . . 117

2.8.4 Meßtheoretische Grundlagen . . . . . . . . . . 118 
2.8.5 Die Programmessung . . . . . . . . . . . . . . . . 121

2.8.5.1 Meßstrategie . . . . . . . . . . . . . 121

2.8.5.2 Validation von Programmaßen . . . . . . . . . 123

2.8.6 Meß-Tools . . . . . . . . . . . . . . . . . . 124

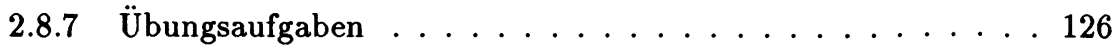

2.8.8 Quellen- und Literaturhinweise . . . . . . . . . . . . . 127

3 Programmieren im Großen $\quad 129$

3.1 Software-Management . . . . . . . . . . . . . . 130

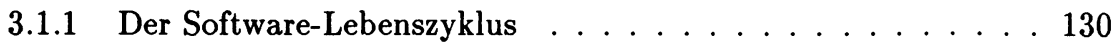

3.1.1.1 Die Software-Entwicklungsphasen . . . . . . . 130

3.1.1.2 Sequentielle Software-Lebenszyklusmodelle . . . . . 140

3.1.1.3 Nichtsequentielle Software-Lebenszyklusmodelle . . 142

3.1.1.4 Übungsaufgaben . . . . . . . . . . . . . . . . . . . 147

3.1.1.5 Quellen- und Literaturhinweise . . . . . . . . . . 148

3.1.2 Managementformen . . . . . . . . . . . . . 150

3.1.2.1 Projektmanagement . . . . . . . . . . . 150

3.1.2.2 Konfigurationsmanagement . . . . . . . . . . . 155

3.1.2.3 Software-Qualitätsmanagement . . . . . . . . . 156

3.1.2.4 CASE-Tool-Einsatz . . . . . . . . . . . . 161

3.1.2.5 Übungsaufgaben . . . . . . . . . . . . . . . . . . . . 164

3.1.2.6 Quellen- und Literaturhinweise . . . . . . . . . . . 164

3.2 Klassische Entwurfsmethoden . . . . . . . . . . . . . . 167

3.2.1 Entscheidungstabellen . . . . . . . . . . . . . . 167

3.2.1.1 Grundlegende Eigenschaften . . . . . . . . . 167

3.2.1.2 Einfache Beispiele . . . . . . . . . . . . . . 168

3.2.1.3 Übungsaufgaben . . . . . . . . . . . . . . . . 170

3.2.1.4 Quellen- und Literaturhinweise . . . . . . . . . . . 171

3.2.2 Petri-Netze . . . . . . . . . . . . . . . . . . . 173

3.2.2.1 Grundeigenschaften . . . . . . . . . . 173

3.2.2.2 Netztransformationen . . . . . . . . . . . . . 175

3.2.2.3 Petri-Netz-Interpretationen . . . . . . . . . 176 
3.2.2.4 Übungsaufgaben . . . . . . . . . . . . . . 178

3.2.2.5 Quellen- und Literaturhinweise . . . . . . . . . . 178

3.2.3 Das Entity-Relationship-Modell . . . . . . . . . . . . . . . 180

3.2.3.1 Darstellungsform . . . . . . . . . . . . . . . . 180

3.2.3.2 Relationsbeschreibungen . . . . . . . . . . 182

3.2.3.3 Übungsaufgaben . . . . . . . . . . . . . . . . . . 184

3.2.3.4 Quellen- und Literaturverzeichnis . . . . . . . . 185

3.2.4 Die SA-Methode . . . . . . . . . . . . . . . . . 187

3.2.4.1 Überblick . . . . . . . . . . . . . . . . . . . . . 187

3.2.4.2 Entwurfsdokumente . . . . . . . . . . . 188

3.2.4.3 Übungsaufgaben . . . . . . . . . . . . . . . . . . 192

3.2.4.4 Quellen- und Literaturhinweise . . . . . . . . . . 193

3.3 Formale Spezifikation . . . . . . . . . . . . . . . . . . . 195

3.3.1 Einleitung . . . . . . . . . . . . . . . . . . 195

3.3.2 Formale Spezifikation mittels VDM . . . . . . . . . . . . 197

3.3.2.1 Einführende Beispiele . . . . . . . . . . . 197

3.3.2.2 Grundkonstrukte der Spezifikationssprache . . . . 198

3.3.2.3 Die VDM-Spezifikationsmethodik . . . . . . . 206

3.3.2.4 Übungsaufgaben . . . . . . . . . . . . . . . . 208

3.3.2.5 Quellen- und Literaturhinweise . . . . . . . . . . 209

3.3.3 Formale Spezifikation mit LOTOS . . . . . . . . . . . . 211

3.3.3.1 Das Grundkonzept von LOTOS . . . . . . . . . 211

3.3.3.2 Basis-LOTOS . . . . . . . . . . . . . . . . 214

3.3.3.3 Datentypen . . . . . . . . . . . . . . . . . . 219

3.3.3.4 Das erweiterte LOTOS . . . . . . . . . . . 225

3.3.3.5 Softwareentwicklung mit LOTOS . . . . . . . . . 231

3.3.3.6 Übungsaufgaben . . . . . . . . . . . . . . . . 236

3.3.3.7 Quellen- und Literaturhinweise . . . . . . . . . . 237

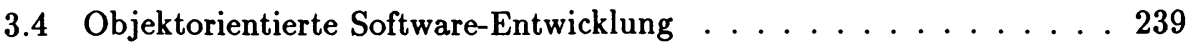

3.4.1 Allgemeine Grundlagen . . . . . . . . . . . . . 239

3.4.2 Quellen- und Literaturhinweise . . . . . . . . . . . 245 
3.5 Objektorientierte Analyse nach COAD/ YOURDON . . . . . . . . . 247

3.5.1 Methodische Grundlagen . . . . . . . . . . . . . . 247

3.5.2 Identifikation der Klassen und Objekte . . . . . . . . . . . . . 249

3.5.3 Herleiten der Objektstruktur ... . . . . . . . . . 250

3.5.4 Identifikation der Subjektstruktur ............ 251

3.5.5 Ergänzung der Objekte um Attribute . . . . . . . . . 252

3.5.6 Erweiterung der Objekte um Dienste ........... 253

3.5.7 Dokumentation .................. 255

3.5.8 Das OOA-Modell . . . . . . . . . . . . . 255

3.5 .9 Übungsaufgaben . . . . . . . . . . . . . . . 256

3.5.10 Quellen- und Literaturhinweise . . . . . . . . . . . . . 257

3.6 Objektorientierter Entwurf nach COAD/ YOURDON . . . . . . . 259

3.6.1 Methodische Grundlagen . . . . . . . . . . . . 259

3.6.2 Entwurf der Problembereichskomponente . . . . . . . . 260

3.6.3 Entwurf der Nutzerschnittstelle . . . . . . . . . . . 261

3.6.4 Entwurf des Task-Management . . . . . . . . . . . 261

3.6.5 Entwurf des Daten-Management . . . . . . . . . . 262

3.6 .6 Übungsaufgaben ................... 262

3.6.7 Quellen- und Literaturhinweise . . . . . . . . . . . 262

3.7 Objektorientierte Programmierung . . . . . . . . . . . . . . 264

3.7.1 Objektorientierte Programmierung in Smalltalk . . . . . . 264

3.7.2 Objektorientierte Programmierung in $\mathrm{C}++\ldots . . . . .267$

3.7.3 Objektorientierte Programmgenerierung . . . . . . . . 268

3.7 .4 Übungsaufgaben . . . . . . . . . . . . . . . . . 269

3.7.5 Quellen- und Literaturhinweise . . . . . . . . . . . 269

3.8 Ein einfaches Beispiel . . . . . . . . . . . . . . 271

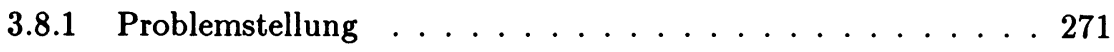

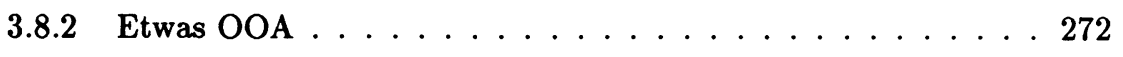

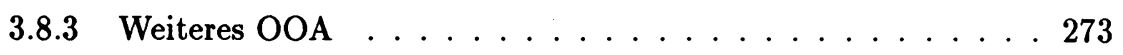

3.8.4 Die OOD-Problembereichskomponente . . . . . . . . 276

3.8.5 Bereits etwas OOP in Smalltalk . . . . . . . . . . . 277

3.8 .6 OOP in $\mathrm{C}++\ldots \ldots . \ldots 281$

3.8.7 Die OOD-Nutzerschnittstelle (HIC) . . . . . . . . 282 
4 Lösungshinweise zu den Übungen 285

4.1 Programmieren im Kleinen . . . . . . . . . . . . . . 285

4.1.1 Strukturierten Programmierung. . . . . . . . . . . 285

4.1.2 Modularisierung . . . . . . . . . . . . . . 287

4.1.3 Makroprogrammierung . . . . . . . . . . . 287

4.1 .4 Programmtest . . . . . . . . . . . . . 288

4.1.5 Programmodifikation ................ 289

4.1.6 Programmdokumentation . . . . . . . . . . . . 290

4.1 .7 Programmessung . . . . . . . . . . . . . . . . 292

4.2 Programmierung im Großen . . . . . . . . . . . . . . . . . 293

4.2.1 Software-Lebenszyklus ............... 293

4.2 .2 Managementformen ................ 294

4.2.3 Entscheidungstabellen ................ 295

4.2 .4 Petri-Netze .................... 296

4.2.5 Entity-Relationship-Modell . . . . . . . . . . . 297

4.2.6 Strukturierte Analyse . . . . . . . . . . . . . 298

4.2.7 Formale Spezifikation mit VDM . . . . . . . . . . . 300

4.2.8 Formale Spezifikation mit LOTOS . . . . . . . . . . . 301

4.2 .9 Objektorientierte Analyse . . . . . . . . . . . . 302

4.2.10 Objektorientierter Entwurf . . . . . . . . . . . . 304

4.2.11 Objektorientierte Programmierung . . . . . . . . . . 305

$\begin{array}{ll}\text { Sachwortverzeichnis } & \mathbf{3 0 7}\end{array}$ 